\title{
BOREL PARAMETRIZATIONS
}

\author{
BY \\ R. DANIEL MAULDIN ${ }^{1}$
}

\begin{abstract}
Let $X$ and $Y$ be uncountable Polish spaces and $B$ a Borel subset of $X \times Y$ such that for each $x, B_{x}$ is uncountable. A Borel parametrization of $B$ is a Borel isomorphism, $g$, of $X \times E$ onto $B$ where $E$ is a Borel subset of $Y$ such that for each $x, g(x, \cdot)$ maps $E$ onto $B_{x}=\{y:(x, y) \in B\}$. It is shown that $B$ has a Borel parametrization if and only if $B$ contains a Borel set $M$ such that for each $x, M_{x}$ is a nonempty compact perfect set, or, equivalently, there is an atomless conditional probability distribution, $\mu$, so that for each $x, \mu\left(x, B_{x}\right)>0$. It is also shown that if $Y$ is dense-in-itself and $B_{x}$ is not meager, for each $x$, then $B$ has a Borel parametrization.
\end{abstract}

Let $G$ be a Borel subset of the product $X \times Y$ of two Polish spaces. A Borel parametrization of $B$ is a Borel isomorphism $g$ of $X \times E$, where $E$ is a Borel subset of $Y$ onto $B$ such that for each $x, g(x, \cdot)$ maps $E$ onto $B_{x}=\{y$ : $(x, y) \in B\}$. Of course, if $B$ has a parametrization, then all the sets, $B_{x}$, must have the same cardinality. If $X$ is countable, then this cardinality condition on the sets $B_{x}$ is necessary and sufficient for $B$ to have a Borel parametrization. For in case $X$ is countable, fix a Borel subset $E$ of $Y$ of the correct cardinality and fix, for each $x$ in $X$, a Borel isomorphism $\theta_{x}$ of $E$ onto $B_{x}$ and then define $g$ by $g(x, y)=\theta_{x}(y)$. It will be assumed throughout this paper that $X$ and $Y$ are uncountable Polish spaces. It is easy to see that when the sets $B_{x}$ are uncountable, one can require $E=Y$.

It is an easy corollary of a theorem of Lusin that if each $B_{x}$ is countably infinite or if each $B_{x}$ has cardinality $n$, some fixed integer $n$, then $B$ has a Borel parametrization. Let us note that Choksi [4] in his investigation of measurable transformations on compact groups phrased the parametrization problem as follows.

Let $B$ be a Borel subset of $X \times Y$ with each $B_{x}$ uncountable. Does $B$ have a Borel parametrization?

It was noted by Stone [13] that for Choksi's investigation, Borel parametrizations were not really necessary. In fact, only measurable parametrizations are needed. The author and D. Cenzer have shown that such parametrizations exist [3].

The purpose of this paper is to investigate the existence of Borel parametrizations under the assumption that each $B_{x}$ is uncountable. Various

Received by the editors August 29, 1977.

AMS (MOS) subject classifications (1970). Primary 28A20; Secondary 04A15, 02K30, 54H05.

${ }^{1}$ Research supported in part by NSF Grant MCS 78104376 and a Faculty Development Grant from North Texas State University.

(c) 1979 American Mathematical Society 0002-9947/79/0000-0259/\$04.00 
necessary and sufficient conditions are determined in order that a Borel parametrization exist. One simple formulation is the following theorem.

THEOREM. $A$ Borel subset $B$ of $X \times Y$ with each $B_{x}$ uncountable has a Borel parametrization if and only if $B$ contains a Borel set $M$ such that for each $x, M_{x}$ is a nonempty compact perfect set or equivalently there is a conditional measure distribution, $\mu$, such that for each $x, \mu(x, \cdot)$ is atomless and $\mu\left(x, B_{x}\right)>0$.

It is also shown that if $Y$ is dense-in-itself and each $B_{x}$ is not meager in $Y$, then $B$ has a Borel parametrization.

I. A sufficient condition. Our first goal is to obtain a sufficient condition for the existence of a Borel parametrization.

Theorem 1.1. Let $X$ and $Y$ be uncountable Polish spaces and $B$ a Borel subset of $X \times Y$ such that each $B_{x}$ is a nonempty compact perfect set. Then there is a Borel subset $M$ of $B$ such that each $M_{x}$ is a nonempty perfect set, and a Borel parametrization $k$ of $X \times Y$ onto $M$.

Proof. Let $\{V(n)\}_{n=1}^{\infty}$ be a countable base for the topology of $Y$ consisting of open balls of diameter less than 1. For each pair of integers $(n, m)$ such that $\operatorname{cl}(V(n)) \cap \operatorname{cl}(V(m))=\varnothing$, let $E(n, m)=\Pi_{X}((X \times V(n)) \cap B) \cap \Pi_{X}((X$ $\times V(m)) \cap B)$. It follows from the results of Arsenin [1] and Kunugui [5] that $E(n, m)$ is a Borel set. Since the sets $E(n, m)$ fill up $X$ one can find a sequence $\left\{S_{t}\right\}_{t=1}^{\infty}$ of disjoint Borel sets and a map, $\theta$, of $N$, the natural numbers, into $N \times N$ such that (1) $\cup S_{t}=X$ and (2) for each $t, S_{t} \subset E(\theta(t))$ $=E\left(\theta_{1}(t), \theta_{2}(t)\right)$.

Set

$$
M(\langle 0\rangle)=\bigcup_{t \in N} \operatorname{cl}_{y}\left(\left(S_{t} \times V\left(\theta_{1}(t)\right)\right) \cap B\right) .
$$

Here we use the following notation. If $E \subset X \times Y$, then $\mathrm{cl}_{y}(E)=\{(x, y)$ : $(x, y)$ is a cluster point of $\left.\{x\} \times E_{x}\right\}$. It follows from the results of Arsenin and Kunugui that if $E$ is a Borel subset of $X \times Y$ and each $E_{x}$ is both a $K_{\sigma}$ set and a $G_{\delta}$ set, then $\mathrm{cl}_{y}(E)$ is a Borel set. Of course, a $K_{\sigma}$ set is a set which can be expressed as the union of countably many compact sets. Thus, $M(\langle 0\rangle)$ is a Borel set. Similarly,

$$
M(\langle 1\rangle)=\bigcup_{t \in N} \mathrm{cl}_{y}\left(\left(S_{t} \times V\left(\theta_{2}(t)\right)\right) \cap B\right)
$$

is a Borel set.

Notice that $M(\langle 0\rangle)$ and $M(\langle 1\rangle)$ are subsets of $B$ and for each $x, M(\langle 0\rangle)_{x}$ and $M(\langle 1\rangle)_{x}$ are disjoint nonempty compact perfect sets with diameter less than 1 .

Continuing by induction, one finds that there is a family

$$
\left\{M\left(\left\langle e_{1}, \ldots, e_{n}\right\rangle\right): e_{i}=0 \text { or } 1 \text { and } n \text { is a positive integer }\right\}
$$


such that for each $\left\langle e_{1}, \ldots, e_{n}\right\rangle$

(1) $M\left(\left\langle e_{1}, \ldots, e_{n}\right\rangle\right)$ is a Borel set,

(2) for each $x,\left(M\left(\left\langle e_{1}, \ldots, e_{n}\right\rangle\right)\right)_{x}$ is a nonempty compact perfect set with diameter less than $2^{-n}$,

(3) if $\left\langle e_{1}, \ldots, e_{n}\right\rangle \neq\left\langle e_{1}^{\prime}, \ldots, e_{n}^{\prime}\right\rangle$, then

$$
M\left(\left\langle e_{1}, \ldots, e_{n}\right\rangle\right) \cap M\left(\left\langle e_{1}^{\prime}, \ldots, e_{n}^{\prime}\right\rangle\right)=\varnothing,
$$

(4) if $e=0$ or 1 ,

$$
M\left(\left\langle e_{1}, \ldots, e_{n}, e\right\rangle\right) \subset M\left(\left\langle e_{1}, \ldots, e_{n}\right\rangle\right) .
$$

For each $n$, set

$$
T_{n}=\bigcup\left\{M\left(\left\langle e_{1}, \ldots, e_{n}\right\rangle\right): e_{i}=0 \text { or } 1\right\}
$$

and set

$$
M=\bigcap_{n=1}^{\infty} T_{n} .
$$

Clearly, $M$ is a Borel set, $M \subset B$ and each $M_{x}$ is a nonempty perfect set.

For each $e=\left\{e_{n}\right\}_{n=1}^{\infty} \in\{0,1\}^{N}$ and $x \in X$, set $f(x, e)=(x, y)$, where

$$
\{y\}=\left(\bigcap_{n=1}^{\infty} M\left(\left\langle e_{1}, \ldots, e_{n}\right\rangle\right)\right)_{x} .
$$

Clearly, $f$ is a one-to-one map of $X \times C$, where $C=\{0,1\}^{N}$, onto $M$ and for each $x, f(x, \cdot)$ maps $C$ onto $M_{x}$. Also, setting

$$
C\left(\left\langle i_{1}, \ldots, i_{n}\right\rangle\right)=\left\{e \in C: e_{p}=i_{p}, 1<p<n\right\}
$$

we have

$$
f\left(X \times C\left\langle i_{1}, \ldots, i_{n}\right\rangle\right)=M\left(\left\langle i_{1}, \ldots, i_{n}\right\rangle\right) \cap M .
$$

But this implies $f^{-1}$ is a Borel map of $M$ onto $X \times C$. According to a theorem of Lusin and Souslin [6, p. 489], $f$ is a Borel isomorphism. Let $h$ be a Borel isomorphism of $Y$ onto $C$ and set $k(x, y)=f(x, h(y))$. Clearly, $k$ is a Borel parametrization of $M$.

THEOREM 1.2. Let $X$ and $Y$ be uncountable Polish spaces, $B$ a Borel subset of $X \times Y$ and $M$ a Borel set lying in $X \times Y$ such that there is a Borel parametrization, $k$, of $X \times Y$ onto $M$. Then $B$ has a Borel parametrization.

Proof. A Schoeder-Bernstein type argument is given for this theorem. This argument is also used in [3].

Set $S_{0}=B-M$ and $T_{0}=X \times Y-B$. Thus,

$$
\begin{aligned}
X \times Y & =M \cup S_{0} \cup T_{0} \\
& =T_{0} \cup S_{0} \cup\left(T_{1} \cup S_{1}\right) \cup \cdots \cup\left(T_{n} \cup S_{n}\right) \cup \cdots \cup D,
\end{aligned}
$$


where $T_{n}=k^{n}\left(T_{0}\right), S_{n}=T^{n}\left(S_{0}\right)$ and $D=\bigcap_{p=1}^{\infty} k^{p}(B)$. We also have

$$
\begin{aligned}
B & =M \cup S_{0} \\
& =S_{0} \cup\left(T_{1} \cup S_{1}\right) \cup \cdots \cup\left(T_{n} \cup S_{n}\right) \cup \cdots \cup D \\
& =\left(T_{1} \cup S_{0}\right) \cup\left(T_{2} \cup S_{1}\right) \cup \cdots \cup\left(T_{n+1} \cup S_{n}\right) \cup \cdots \cup D .
\end{aligned}
$$

Set $H=D \cup\left(\cap_{n=0}^{\infty} S_{n}\right)$ and $G=\bigcap_{n=0}^{\infty} T_{n}$ and define

$$
g(x, y)= \begin{cases}(x, y), & \text { if }(x, y) \in H, \\ k(x, y), & \text { if }(x, y) \in G .\end{cases}
$$

It can easily be checked that $g$ is a one-to-one map of $X \times Y$ onto $B$. Also, since all the sets $S_{n}, T_{n}$ and $D$ are Borel sets, $g$ is a Borel isomorphism. Finally, since $g(x, \cdot)$ maps $Y$ onto $B_{x}, g$ is a Borel parametrization of $B$. Q.E.D.

As an immediate corollary of Theorems 1.1 and 1.2, we have the following theorem.

THEOREM 1.3. If $B$ is a Borel subset of $X \times Y$ and $B$ contains a Borel set $M$ such that each $M_{x}$ is a nonempty compact perfect set, then $B$ has a Borel parametrization.

Let us note that Theorem 1.3 improves a theorem of Larman [7], since the existence of a parametrization of $B$ is a stronger statement that the existence of $2^{\mathrm{K}_{0}}$ disjoint uniformizations.

II. Borel sets with large sections. In this section, it is shown that if $B$ is a Borel subset of $X \times Y$ such that each $B_{x}$ is uncountable, then $B$ has a Borel parametrization if and only if each section of $B$ is large in a quantitative sense. The instrument for measuring the quantitative size of the sections of a set is the conditional measure distribution. With the aid of this instrument a descriptive characterization of those Borel sets $B$ having a Borel parametrization is given. It is simply that $B$ contains a Borel set $M$ each section of which is a nonempty compact perfect set. All this is formulated in Theorem 2.4 .

It is also shown in Theorem 2.8 that $B$ has a Borel parametrization if each section of $B$ is large in a qualitative sense: each $B_{x}$ is not meager. This seems slightly unusual in that measure and category are in some sense dual properties [9].

A conditional measure distribution on $X \times \mathscr{B}(Y)$ is a map $\mu$ from $X \times$ $\mathscr{B}(Y)$ into the nonnegative real numbers such that (1) for each $x, \mu(x, \cdot)$ is a measure on $\mathscr{B}(Y)$ and (2) for each $E$ in $\mathscr{B}(Y), \mu(\cdot, E)$ is a Borel measurable function on $X$. The standard Borel field on $Y$ is denoted by $\mathscr{B}(Y)$.

A useful fact concerning these distributions is the following theorem. 
THEOREM 2.1. Let $\mu$ be a conditional measure distribution on $X \times \mathscr{B}(Y)$. If $B$ is a Borel subset of $X \times Y$, then $f(x)=\mu\left(x, B_{x}\right)$ is a Borel measurable function on $X$ and

$$
\nu(x, E)=\mu\left(x, E \cap B_{x}\right)
$$

is a conditional measure distribution on $X \times \mathscr{B}(Y)$.

Proof. Let $W$ be the family of all Borel subsets of $X \times Y$ for which the theorem holds.

If $B$ is a Borel set of the form $S \times T$ and $\mu$ is a conditional measure distribution, $f(x)=\mu\left(x, B_{x}\right)=\mu\left(x,(S \times T)_{x}\right)=\mu(x, T) \chi_{s}$, where $\chi_{s}$ is the characteristic function of the Borel set $S$. Thus, $f$ is Borel measurable. Clearly, for each $x, \nu(x, \cdot)$ is a measure on $B(Y)$, where $\nu(x, E)=\mu\left(x, E \cap B_{x}\right)$. Thus, $W$ contains all Borel sets of the form $S \times T$.

It can be easily checked that $W$ is closed under monotone limits and finite disjoint unions. It follows that $W$ is the family of all Borel subsets of $X \times Y$. Q.E.D.

THEOREM 2.2. Let $\mu$ be a conditional measure distribution on $X \times \mathscr{B}(Y)$ and let $B$ be a Borel subset of $X \times Y$. Then for each $\theta, 0<\theta<1$, there is a Borel subset $M$ of $X \times Y$ such that $M \subseteq B$, and for every $x, M_{x}$ is a compact set and $\mu\left(x, M_{x}\right)>\theta \mu\left(x, B_{x}\right)$.

Proof. Let $\varphi$ be an embedding of $Y$ into $H$, the Hilbert cube. Define $\hat{\mu}$ on $X \times \mathscr{B}(H)$ by $\hat{\mu}(x, E)=\mu\left(x, \varphi^{-1}(E)\right)$. Then $\hat{\mu}$ is a conditional measure distribution on $X \times \mathscr{B}(H)$ and (id $\times \varphi)(B)=\hat{B}$ is a Borel subset of $X \times H$. Thus, in order to prove the theorem it suffices to prove it in the case that $\mu$ is defined on $X \times \mathscr{B}(H)$.

Let $W$ be the family of all Borel subsets of $X \times H$ for which the conclusion of the theorem holds for all conditional measure distributions.

Let $B_{1}$ and $B_{2}$ belong to $W$, let $\mu$ be a conditional measure distribution and let $0<\theta<1$. Set

$$
\mu_{1}(x, E)=\mu\left(x, E \cap B_{1 x}\right) \text { and } \mu_{2}(x, E)=\mu\left(x, E \cap\left(B_{2}-B_{1}\right)_{x}\right) .
$$

According to Theorem 2.1, $\mu_{1}$ and $\mu_{2}$ are conditional measure distributions. Let $M_{1}$ and $M_{2}$ be Borel sets with compact sections so that $M_{i} \subset B_{i}$ and $\mu_{i}\left(x, M_{i x}\right) \geqslant \theta \mu_{i}\left(x, B_{i x}\right)$, for $i=1,2$. Set $M=M_{1} \cup M_{2}$. Clearly $M$ is a Borel set with compact sections. Also,

$$
\begin{aligned}
\mu\left(x, M_{\dot{x}}\right) & =\mu\left(x,\left(M \cap B_{1}\right)_{x}\right)+\mu\left(x,\left(M \cap\left(B_{2}-B_{1}\right)\right)_{x}\right) \\
& \geqslant \mu\left(x,\left(M_{1} \cap B_{1}\right)_{x}\right)+\mu\left(x,\left(M_{2} \cap\left(B_{2}-B_{1}\right)\right)_{x}\right) \\
& \geqslant \mu_{1}\left(x, M_{1 x}\right)+\mu_{2}\left(x, M_{2 x}\right) \\
& \geqslant \theta \mu\left(x, B_{1 x}\right)+\theta \mu\left(x,\left(B_{2}-B_{1}\right)_{x}\right) \\
& \geqslant \theta \mu\left(x,\left(B_{1} \cup B_{2}\right)_{x}\right) .
\end{aligned}
$$


From this it follows that $W$ is closed under finite unions. We will now show that $W$ is a monotone family.

First, suppose $\left\{S_{n}\right\}$ is an increasing sequence from $W$ and $S=\cup S_{n}$. For each $n$, let $K_{n}$ be a Borel set with compact sections so that $K_{n} \subset S_{n}$ and $\mu\left(x, K_{n x}\right) \geqslant \sqrt{\theta} \cdot \mu\left(x, S_{n x}\right)$. For each $n$, let $M_{n}=\cup\left\{K_{p}: p<n\right\}$. For each $n$, let $T_{n}=\left\{x: \mu\left(x, M_{n x}\right) \geqslant \theta \mu\left(x, S_{x}\right)\right\}$. According to Theorem 2.1, the map $h_{n}(x)=\mu\left(x, M_{n x}\right)-\theta \mu\left(x, S_{x}\right)$ is a Borel measurable map of $X$ into $R$. Thus, $T_{n}$ is a Borel set for each $n$. Let $M$ be the Borel subset of $X \times H$ such that $M_{x}=M_{n x}$, where $n$ is the first integer so that $x \in T_{n}$. The set $M$ has the required properties and $S \in W$.

Now, suppose $S_{n}$ is a decreasing sequence from $W$ and $S=\cap S_{n}$.

Let $\lambda$ map $X \times \mathscr{B}(H)$ into the real numbers by

$$
\lambda(x, B)= \begin{cases}\mu\left(x, S_{x} \cap B\right) / \mu\left(x, S_{x}\right), & \text { if } \mu\left(x, S_{x}\right)>0, \\ 0, & \text { if } \mu\left(x, S_{x}\right)=0 .\end{cases}
$$

Then $\lambda$ is a conditional measure distribution on $X \times \mathscr{B}(H)$. For each $n$, let $K_{n}$ be a Borel set with compact sections so that $K_{n} \subseteq S_{n}$ and $\lambda\left(x, K_{n x}\right)>$ $\theta_{n} \lambda\left(x, S_{n x}\right)$, where $\theta_{n}=1-(1-\theta) / 2^{n}$. Let $M=\cap K_{n}$. Then $M$ has compact sections and $M \subset S$. Also, if $\mu\left(x, S_{x}\right)>0$

$$
\lambda\left(x, M_{x}\right) \geqslant 1-\sum\left(1-\theta_{n}\right)=\theta .
$$

Thus, for all $x, \mu\left(x, M_{x}\right) \geqslant \theta \mu\left(x, S_{x}\right)$. The set $M$ has the required properties and $S \in W$.

Finally, since $W$ clearly contains the sets of the form $A \times K$, where $A$ is Borel in $X$ and $K$ is compact, $W$ is the family of all Borel subsets of $X \times H$. Q.E.D.

Theorem 2.3. Let $X$ and $Y$ be Polish spaces, let $\mu$ be a conditional measure distribution on $X \times \mathscr{B}(Y)$ such that for each $x, \mu(x, \cdot)$ is an atomless nontrivial measure, and let $B$ be a Borel subset of $X \times Y$ such that for each $x$ in $X$, $\mu\left(x, B_{x}\right)>0$. For each $\theta, 0<\theta<1$, there is Borel subset $M$ of $B$ such that for each $x, \mu\left(x, M_{x}\right) \geqslant \theta \mu\left(x, B_{x}\right)$ and $M_{x}$ is a compact perfect set. Moreover, $B$ has a Borel parametrization.

Proof. Let $\theta$ be a number between 0 and 1 . According to Theorem 2.2 there is a Borel subset $K$ of $B$ such that for each $x, K_{x}$ is compact and $\mu\left(x, K_{x}\right) \geqslant \theta \mu\left(x, B_{x}\right)$. Let $M=\{(x, y)$ : if $V$ is a neighborhood of $y$, then $\left.\mu\left(x, K_{x} \cap V\right)>0\right\}$. Clearly, $M \subset K$ and for each $x, M_{x}$ is a compact perfect set such that $\mu\left(x, M_{x}\right)=\mu\left(x, K_{x}\right)$.

To see that $M$ is a Borel set, let $\left\{V_{n}\right\}_{n=1}^{\infty}$ be a base for the topology of $Y$ and let $T_{n}=\left\{x: \mu\left(x, K_{x} \cap V_{n}\right)=0\right\}$. According to Theorem 2.1, $T_{n}$ is a 
Borel set. Also,

$$
(X \times Y)-M=\bigcup_{n=1}^{\infty}\left(T_{n} \times V_{n}\right)
$$

The fact that $B$ has a Borel parametrization now follows from Theorem 1.3. Q.E.D.

Theorem 2.4. Let $X$ and $Y$ be uncountable Polish spaces and let $B$ be a Borel subset of $X \times Y$ such that for each $x, B_{x}$ is uncountable. The following statements are equivalent

1. $B$ has a Borel parametrization.

2. There is a conditional probability distribution $\mu$ on $X \times \mathscr{B}(Y)$ such that for each $x, \mu\left(x, B_{x}\right)=1$ and for each $x, \mu(x, \cdot)$ is atomless.

3. $B$ contains a Borel set $M$ such that for each $x, M_{x}$ is a nonempty compact perfect set.

Proof. Assume statement 1 holds and let $g$ be a one-to-one Borel map of $X \times Y$ onto $B$ which parametrizes $B$. Let $f$ be a Borel isomorphism of $I$, the unit interval, onto $Y$. Set

$$
\mu(x, E)=\lambda\left(f^{-1}\left(\left(g^{-1}(B \cap(X \times E))\right)_{x}\right)\right)
$$

for each $x$ in $X$ and $E$ in $B(Y)$, where $\lambda$ is Lebesgue measure on $I$.

Clearly, for each $x, \mu(x, \cdot)$ is an atomless probability measure on $\mathscr{B}(Y)$ such that $\mu\left(x, B_{x}\right)=1$. From the definition of $\mu$ and from Theorem 2.1 we see that for each $E$ in $\mathscr{B}(Y), \mu(\cdot, E)$ is a Borel measurable function. Thus, statement 1 implies statement 2.

Theorem 2.3 shows that statement 2 implies statement 3 . Theorem 1.3 shows that statement 3 implies statement 1. Q.E.D.

Blackwell and Ryll-Nardzewski [2] showed that under the hypothesis of Theorem 2.3, $B$ has a Borel uniformization. In other words, there is a Borel measurable map $f$ of $X$ into $Y$ whose graph is a subset of $B$. Actually, $B$ may be filled up by such graphs as the next theorem shows.

THEOREM 2.5. Assume that the subset $B$ of $X \times Y$ has a Borel parametrization. Then $B$ is filled up by $2^{\aleph_{0}}$ disjoint Borel uniformizations.

Proof. Let $g: X \times Y \rightarrow B$ be a Borel parametrization of $B$. For each $y \in Y$, let $f_{y}(x)=\Pi_{Y}(g(x, y))$. Then the graphs of the functions $f_{y}$ are disjoint Borel uniformizations.

There are results similar to those just obtained where category is taken as an indicator of largeness instead of measure. However, to prove that if each section of $B$ is not meager, then $B$ contains a Borel set $M$ such that each $M_{x}$ is a nonempty compact perfect set seems to require some more machinery. Let us make the following notation. 
Seq denotes the set of all finite sequence of positive integers: Seq $=$ $\cup_{k=1}^{\infty} N^{k} .\{0,1\}^{*}$ denotes the set of all finite sequences of zeros and ones. If $s=\left(k_{1}, \ldots, k_{p}\right) \in$ Seq and $n \in N$, then $s * n=\left(k_{1}, \ldots, k_{p}, n\right)$. If $s \in$ Seq, then $\operatorname{lh}(s)$ denotes the length of the sequence $s$.

If $B \subset X \times Y$ and $U \subset Y$, then $B_{U}^{*}=\left\{x: B^{x} \cap U\right.$ is comeager in $\left.U\right\}$. If $B$ is Borel and $U$ is open, $B_{U}^{*}$ is a Borel set [15].

We will need the following theorem of $\mathrm{H}$. Sarbadhikari [11].

TheOREM 2.6. Let $X$ and $Y$ be Polish spaces and $B$ a Borel subset of $X \times Y$. There is a sequence $\left\{Z_{k}\right\}_{k=1}^{\infty}$ of Borel subsets of $X \times Y$ such that

(a) $\cap Z_{k} \subset B$,

(b) given any nonempty open set $W$ in $Y$, any $k$ and any $\varepsilon>0$, there is $a$ Borel set $F, F \subset Z_{k} \cap(X \times W)$ such that for all $x, F_{x}$ is closed, $\operatorname{diam}\left(F_{x}\right)<\varepsilon$ and if $x \in B_{X}^{*}$, then $F_{x}$ is not meager.

Theorem 2.7. Let $Y$ be a dense-in-itself Polish space, $B$ a Borel subset of $X \times Y$ such that for each $x, B_{x}$ is comeager in $Y$ and $\left\{Z_{k}\right\}_{k=1}^{\infty}$ a sequence of Borel sets so that Theorem 2.6 holds. Then there is a family $\{D(s): s \in$ Seq $\}$ of Borel subsets of $X, a$ family $\left\{V(s, e): s \in \operatorname{Seq}, e \in\{0,1\}^{*}\right.$ and $\left.\ln (s)=\operatorname{lh}(e)\right\}$ of open subsets of $Y$, and a sequence $\left\{M_{n}\right\}$ of Borel subsets of $X \times Y$ such that

(i) $D(s) \cap D(t)=\varnothing$, if $s, t \in N^{n}$ and $s \neq t$;

(ii) $D(s)=\cup\{D(s * n): n \in N\}$ and $X=\cup\{D(\langle n\rangle): n \in N\}$;

(iii) for each $x \in D(s),\left(M_{\mathrm{lh}(s)} \cap B\right)_{x} \cap V(s, e)$ is comeager in $V(s, e)$, for each $e \in\{0,1\}^{\mathrm{lh}(s)}$;

(iv) $\overline{V(s, e)} \cap \overline{V\left(s, e^{\prime}\right)}=\varnothing$, if $e \neq e^{\prime}$;

(v) $\operatorname{diam}(V(s, e))<2^{-\ln (s)}$;

(vi) $\overline{V(s * m, e * i)} \subset V(s, e)$;

(vii) if $x \in D(s)$, then $M_{\operatorname{lh}(s) x}$ is a closed set lying in $\cup\{\overline{V(s, e)}: e \in$ $\left.\{0,1\}^{\operatorname{lh}(s)}\right\}$;

(viii) $M_{n+1} \subset M_{n} \subset Z_{n}$.

Proof. Let $U(0)$ and $U(1)$ be nonempty disjoint open subsets of $Y$. Let $F(0)$ and $F(1)$ be Borel subsets of $X \times Y$ such that for $i=0$ or 1 ,

$$
F(i) \subset Z_{1} \cap(X \times Y(i))
$$

and for each $x, F(i)_{x}$ is closed and not meager. The existence of these sets is guaranteed by Theorem 2.6. For each pair $\left(p_{0}, p_{1}\right) \in N^{2}$ such that $\operatorname{cl}\left(V\left(p_{i}\right)\right)$ $\subset U(i)$, and $\operatorname{diam}\left(V\left(p_{i}\right)\right)<2^{-1}$, for $i=0$ or 1 , let

$$
H\left(\left(p_{0}, p_{1}\right)\right)=(F(i) \cap B)_{V\left(p_{0}\right)}^{*} \cap(F(i) \cap B)_{V\left(p_{1}\right)}^{*}
$$

Since the Borel sets $H\left(\left(p_{0}, p_{1}\right)\right)$ cover $X$, there is a sequence $\{D(\langle n\rangle): n \in N\}$ of pairwise disjoint Borel sets and a map $\theta: N \rightarrow N^{2}$ so that for each $n$, $D(\langle n\rangle) \subset H(\theta(n))$. For each $n$, let $V(\langle n\rangle,\langle i\rangle)=V\left(p_{i}\right), i=0$ or 1 , where 
$\theta(n)=\left(p_{0}, p_{1}\right)$. Finally, set

$$
M_{1}=\cup\{D(\langle n\rangle) \times \overline{V(\langle n\rangle,\langle i\rangle)} \cap F(i): n \in N, i=0,1\} .
$$

This completes the construction for $n=1$.

The construction process for $n=2$ is similar to that for the first stage except that there is one additional ingredient. This will now be indicated. Fix $n \in N$ and $i=0$ or 1 . Let $F(\langle n\rangle,\langle i\rangle)$ be a Borel set such that

$$
F(\langle n\rangle,\langle i\rangle) \subset Z_{2} \cap(X \times V(\langle n\rangle,\langle i\rangle))
$$

and for each $x, F(\langle n\rangle,\langle i\rangle)_{x}$ is closed and not meager. Thus, for each $x \in D(\langle n\rangle),(F\langle n\rangle,\langle i\rangle) \cap\left(M_{1} \cap B\right)_{x}$ is not meager in $V(\langle n\rangle,\langle i\rangle)$. Since $Y$ is dense-in-itself, for each $x \in D(\langle n\rangle)$, one can find basic open sets $V\left(q_{j}\right)$ so that diam $V\left(q_{j}\right)<2^{-2}, \overline{V\left(q_{j}\right)} \subset V(\langle n\rangle,\langle i\rangle), x \in\left(F\langle n\rangle,\langle i\rangle \cap M_{1} \cap B\right)_{V\left(q_{j}\right)}^{*}$, $j=0$ or 1 , and $\overline{V\left(q_{0}\right)} \cap \overline{V\left(q_{1}\right)}=\varnothing$. The remainder of the construction at the second stage is similar to that of the first stage. Finally, the construction at the stages greater than 2 is similar to that of stage 2. Q.E.D.

THEOREM 2.8. Under the hypothesis of Theorem 2.7, B contains a Borel set $M$ so that for each $x, M_{x}$ is a nonempty compact perfect set.

Proof. Set $M=\cap M_{n}$. Q.E.D.

TheORem 2.9. Let $Y$ be a dense-in-itself Polish space and $B$ a Borel subset of $X \times Y$ such that for each $x, B_{x}$ is not meager. Then $B$ contains a Borel set $M$ such that each $M_{x}$ is a nonempty compact perfect set. Moreover, $B$ has a Borel parametrization.

Proof. Let $\left\{V_{n}\right\}_{n=1}^{\infty}$ be a base for the topology of $Y$. Set $E_{1}=B_{V_{1}}^{*}$ and if $n>1$, set

$$
E_{n}=B_{V_{n}}^{*}-\bigcup_{m<n} B_{V_{M}^{*}}^{*}
$$

For each $n$ set

$$
H_{n}=\left(X-E_{n}\right) \times V_{n} \cup\left(\left(E_{n} \times V_{n}\right) \cap B\right) .
$$

According to Theorem 2.8, there is a Borel set $M_{n} \subseteq H_{n}$ such that for each $x$, $M_{x}$ is a nonempty compact perfect set. Now, set

$$
M=\bigcup_{n=1}\left(M_{n} \cap\left(E_{n} \times V_{n}\right)\right) .
$$

Clearly, $M$ is a Borel set and each section of $M$ is a nonempty compact perfect set. Also, it follows from Theorem 1.3 that $B$ has a Borel parametrization. Q.E.D.

H. Sarbadhikari [11] generalized a result of $R$. Vaught [15] by showing that if $B$ is a Borel subset of $X \times Y$ and for each $x, B_{x}$ is not meager, then $B$ has a Borel uniformization. We have the following theorem. 
THEOREM 2.10. Under the hypothesis of Theorem 2.9, B is filled up by $2^{\alpha_{0}}$ disjoint Borel uniformizations.

III. Examples. The purpose of this section is to present some examples which show that the existence of a Borel parametrization is a fairly stringent condition.

EXAMPLE 3.1. There is a Borel subset $B$ of $I \times I$ such that for each $x$ in $I$, $B_{x}$ is an uncountable $G_{\delta}$ set and yet $B$ does not have a Borel uniformization, much less a Borel parametrization. In fact, there is no analytic set $A$ such that $A \subset B$ and for each $x, A_{x}$ is a nonempty closed set.

Proof. Let $C_{1}$ and $C_{2}$ be disjoint coanalytic subsets of $I$ which cannot be separated by a Borel set [12]. Let $B_{1}\left(B_{2}\right)$ be a Borel subset of $I \times I$ such that the $X$-projection of $B_{1}\left(B_{2}\right)$ is $I-C_{1}\left(I-C_{2}\right)$ and such that each $X$-section of $B_{1}\left(B_{2}\right)$ is an uncountable $G_{\delta}$ set [8].

It is well known that $B$ does not have a Borel uniformization.

Now, assume $A$ is analytic, $A \subset B$ and for each $x, A_{x}$ is a nonempty closed set. Let $E=(I \times I)-B$. Then $E$ and $A$ are disjoint analytic sets. According to a theorem of Saint-Raymond [10], there is a Borel set $H$ such that $A \subset H$, $H \cap E=\varnothing$ and each $H_{x}$ is compact. However, this implies $H \subset B$ and $H$ (and therefore $B$ ) has a Borel uniformization. This contradiction completes the proof of the theorem.

REMARK. The preceding example answers in the negative a question raised by Tanaka [14]. This example also shows that the results of this paper are sharp.

EXAMPLE 3.2. There is a closed subset $B$ of $I \times I$ such that for each $x$ in $I$, $B_{x}$ is a closed uncountable set and yet $B$ does not have a Borel parametrization. In particular, there is no Borel set $M$ so that $M \subset B$ and for each $x, M_{x}$ is a nonempty perfect set.

Proof. Let $A_{1}$ and $A_{2}$ be analytic subsets of $I$ such that $A_{1} \cup A_{2}=I$ and yet there do not exist Borel sets $K_{1}$ and $K_{2}$ so that $K_{i} \subset A_{i}$ and $K_{1} \cup K_{2}=I$. Let $B_{1}$ be a closed subset of $I \times[0,1 / 3]$ so that $B_{1 x}$ is uncountable if and only if $x \in A_{1}$. Let $B_{2}$ be a closed subset of $I \times[2 / 3,1]$ so that $B_{2 x}$ is uncountable if and only if $x \in A_{2}$. Let $B=B_{1} \cup B_{2}$. The existence of such sets is shown in [12]. Assume that the Borel set $B$ has a Borel parametrization $g, g: I \times I \rightarrow B$. Let

$$
E_{1}=\left\{x: \lambda\left(\left(g^{-1}\left(B_{1}\right)\right)_{x}\right)>1 / 3\right\}
$$

and

$$
E_{2}=\left\{x: \lambda\left(\left(g^{-1}\left(B_{2}\right)\right)_{x}\right)>1 / 3\right\},
$$

where $\lambda$ denotes Lebesgue measure. Since $g^{-1}\left(B_{1}\right)$ and $g^{-1}\left(B_{2}\right)$ are Borel sets, it follows from Theorem 2.1, that $E_{1}$ and $E_{2}$ are Borel sets. However, since $g$ 
is a parametrization of $B, E_{1} \subset A_{1}, E_{2} \subset A_{2}$ and $E_{1} \cup E_{2}=I$. This contradiction establishes the example.

It should be remarked that D. G. Larman [7] has shown that if $B$ is a Borel subset of $X \times Y$ such that each $B_{x}$ is uncountable and both a $G_{\delta}$ and a $K_{\sigma}$ subset of $Y$, then $B$ has $\aleph_{1}$ disjoint uniformizations. The problem of the existence of $2^{\mathrm{K}_{0}}$ disjoint uniformizations is still open.

Example 3.3. There are uncountable Polish spaces $X$ and $Y$ and a Borel subset $B$ of $X \times Y$ such that for each $x, B_{x}$ is uncountable and not meager in $Y$ and yet $B$ does not have a Borel parametrization.

Take $X$ to be $I$ and $Y$ to be $[0,1 / 2] \times\{1\}$. Let $H$ be a Borel subset of $I \times[0,1 / 2]$ such that each section of $H$ is uncountable and such that $H$ does not have a Borel parametrization. Take $B=H \cup(X \times\{1\})$.

EXAMPLE 3.4. There are uncountable Polish spaces $X$ and $Y$ and a Borel subset $B$ of $X \times Y$ such that for each $x, B_{x}$ is comeager in $Y$ and yet $B$ does not have a Borel parametrization.

Take $X$ to be $I$ and take $Y$ to be $C \cup T$ where $C$ is the standard Cantor middle third set in the unit interval and $T$ is the set of midpoints of the complementary open intervals in $I-C$. Let $B=(X \times T) \cup H$, where $H$ is a Borel subset of $X \times C$ such that each section of $H$ is uncountable and yet $H$ does not have a Borel uniformization. If $B$ did have a Borel parametrization, then there would be a Borel set $M$ lying in $B$ with each $M_{x}$ nonempty compact perfect set. But, $M$ would be a subset of $H$ and $H$ would have a Borel uniformization.

AcKNOwLedgement. The author would like to thank Shashi Mohan Srivastava of the Indian Statistical Institute for providing the last two examples which illuminated an error in an earlier version of this paper.

\section{REFERENCES}

1. V. Ya. Arsenin, On the nature of the projection of certain B-sets, Izv. Akad. Nauk SSSR Ser. Mat. 4 (1940), 403-410. MR 3, 225.

2. D. Blackwell and C. Ryll-Nardzewski, Non-existence of everywhere proper conditional distributions, Ann. Math. Statist. 34 (1963), 223-225.

3. D. Cenzer and R. D. Mauldin, Measurable parametrizations and selections, Trans. Amer. Math. Soc. 245 (1978), 399-408.

4. J. R. Choksi, Measurable transformations on compact groups, Trans. Amer. Math. Soc. 184 (1973), 101-124.

5. K. Kunugui, Sur un probleme de M. E. Szpilrajn, Proc. Imperial Acad. Sci. Tokyo 16 (1940), 73-78.

6. K. Kuratowski, Topology. Vol. I, Academic Press, New York, 1966.

7. D. G. Larman, Projecting and uniformizing Borel sets with $K_{o}$-sets. II, Mathematika 20 (1973), 233-246.

8. S. Mazurkiewicz and W. Sierpinski, Sur un probleme concernant les fonctions continues, Fund. Math. 6 (1924), 161-169.

9. J. C. Oxtoby, Measure and category, Springer-Verlag, New York, 1971.

10. J. Saint-Raymond, Boréliens à coupes $K_{o}$, Bull. Soc. Math. France 104 (1976), 389-400. 
11. H. Sarabadhikari, Some uniformization results, Tech. Report Math-Stat., Indian Statistical Institute, June 5, 1975.

12. W. Sierpinski, Sur deux complémentaires analytiques non séparables B, Fund. Math. 17 (1931), 296-297.

13. A. H. Stone, Measure theory, Lecture Notes in Math., no. 541, Springer-Verlag, Berlin and New York, 1976, pp. 43-48.

14. H. Tanaka, Some results in the effective descriptive set theory, Publ. Res. Inst. Math. Sci. Kyoto Univ. Ser. A 3 (1967), 11-52.

15. R. Vaught, Invariant sets in topology and logic, Fund. Math 82 (1974), 269-294.

Department of Mathimatics, North Texas State University, Denton, Texas 76203 